\title{
QUADRATIC FORMS AND CHAIN SEQUENCES
}

\author{
ROBERT SEALL AND MARION WETZEL
}

It has been shown that a monotone Hausdorff moment sequence can be characterized by Stieltjes type quadratic forms. This was a result of the theory of chain sequences [4]. In the present paper we shall characterize an "extended" monotone Hausdorff moment sequence by Jacobi type quadratic forms. This will be done by extending the properties of ordinary chain sequences.

The paper is in two sections. In the first section a relation between chain sequences and Jacobi type quadratic forms will be established. The connection between the "extended" monotone Hausdorff moment problem and chain sequences will be discussed in the second section.

1. Jacobi forms and chain sequences. In the theory of positive definite continued fractions a sequence of nonnegative Stieltjes forms is characterized. It is shown that the quadratic form

$$
\sum_{p=1}^{n} x_{p}^{2}-2 \sum_{p=1}^{n-1} a_{p} x_{p} x_{p+1}, \quad n=2,3,4, \cdots
$$

is positive semidefinite if and only if

$$
a_{p}^{2}=\left(1-g_{p-1}\right) g_{p}, 0 \leqq g_{p-1} \leqq 1, \quad p=1,2,3, \cdots \quad\left(g_{0}=0\right) .
$$

This result is an immediate consequence of a more general theorem characterizing positive definite Jacobi fractions.

We shall now state an analogous theorem for Jacobi forms.

THEOREM 1.1. The quadratic forms

$$
\sum_{p=1}^{n}\left(1+b_{p}\right) x_{p}^{2}-2 \sum_{p=1}^{n-1} a_{p} x_{p} x_{p+1}
$$

and

$$
\sum_{p=1}^{n}\left(1-b_{p}\right) x_{p}^{2}-2 \sum_{p=1}^{n-1} a_{p} x_{p} x_{p+1}, \quad n=2,3,4, \cdots,
$$

are positive semidefinite if and only if

$$
a_{p}^{2}=4\left(1-g_{2 p-2}\right)\left(1-g_{2 p-1}\right) g_{2 p-1} g_{2 p}
$$

Received by the editors December 28, 1962 and, in revised form, May 13, 1963. 


$$
\begin{aligned}
b_{p} & =1-2\left(1-g_{2 p-3}\right) g_{2 p-2}-2\left(1-g_{2 p-2}\right) g_{2 p-1}, \\
& =2\left(1-g_{2 p-2}\right)\left(1-g_{2 p-1}\right)+2 g_{2 p-3} g_{2 p-2}-1,
\end{aligned}
$$

where

$$
0 \leqq g_{p-1} \leqq 1, \quad p=1,2,3, \cdots \quad\left(g_{-1}=1, g_{0}=0\right) .
$$

Such sequences shall be called double chain sequences. The $a_{p}^{2}$ and $b_{p}$ shall be referred to as the elements and the $g_{p}$ as the parameters. Double chain sequences arise in the continued fraction solution of the "extended" monotone Hausdorff moment problem [3].

Proof. We designate the determinants associated with the quadratic forms $(1.3)$ and $(1.4)$ by $B_{p}(1)$ and $B_{p}(-1)$. Their recurrence formulas are

$$
B_{p+1}(1)=\left(1+b_{p+1}\right) B_{p}(1)-a_{p}^{2} B_{p-1}(1)
$$

and

$$
\begin{array}{r}
B_{p+1}(-1)=\left(1-b_{p+1}\right) B_{p}(-1)-a_{p}^{2} B_{p-1}(-1), \quad p=0,1,2, \cdots \\
\left(B_{-1}(1)=B_{-1}(-1)=0, B_{0}(1)=B_{0}(-1)=1\right) .
\end{array}
$$

It can be shown that if the $a_{p}^{2}$ and $b_{p}$ are of the forms (1.5) and (1.6), then

$$
B_{p}(1)=2^{p}\left(1-g_{1}\right)\left(1-g_{2}\right) \cdots\left(1-g_{2 p-1}\right)
$$

and

$$
\begin{aligned}
B_{p}(-1)=2^{p} g_{1}\left(1-g_{2}\right) g_{3} \cdots( & \left(1-g_{2 p-2}\right) g_{2 p-1}, \\
& p=1,2,3, \cdots \quad\left(g_{0}=0\right),
\end{aligned}
$$

where the $g_{p}$ are not necessarily between zero and one. These relations follow immediately by an induction on (1.7) and (1.8).

The proof consists of two cases.

Case 1 . The $a_{p}^{2}$ all positive. We consider the necessity part of the proof. The nonnegativity of the forms (1.3) and (1.4) implies that $B_{p}(1) \geqq 0$ and $B_{p}(-1) \geqq 0$. We observe moreover that the $B_{p}(1)$ and $B_{p}(-1)$ are positive by (1.7) and (1.8). Set $b_{1}=1-2 g_{1}$. From (1.9) and (1.10) it follows that $0<g_{1}<1$. Next write

$$
a_{1}^{2}=4\left(1-g_{1}\right) g_{1} g_{2}
$$

and

$$
b_{2}=1-2\left(1-g_{1}\right) g_{2}-2\left(1-g_{2}\right) g_{3} .
$$


Notice that $g_{2} \neq 1$. The formulas (1.9) and (1.10) give

$$
B_{2}(1)=2\left(1-g_{2}\right)\left(1-g_{3}\right) B_{1}(1)
$$

and

$$
B_{2}(-1)=2\left(1-g_{2}\right) g_{3} B_{1}(-1) .
$$

From (1.11), (1.13) and (1.14) we see that $g_{2},\left(1-g_{2}\right)\left(1-g_{3}\right)$ and $\left(1-g_{2}\right) g_{3}$ are each positive. It follows that $0<g_{2}<1$ and $0<g_{3}<1$.

We proceed with the induction. Suppose that $0<g_{2 p-1}<1$ for $p=1,2,3, \cdots, k, k \geqq 2$. Set

$$
a_{k}^{2}=4\left(1-g_{2 k-2}\right)\left(1-g_{2 k-1}\right) g_{2 k-1} g_{2 k},
$$

and

$$
b_{k+1}=1-2\left(1-g_{2 k-1}\right) g_{2 k}-2\left(1-g_{2 k}\right) g_{2 k+1} .
$$

It is necessary to show that $g_{2 k}$ is not equal to one. The following argument is given. Since $B_{k+1}(1)>0$, using (1.9), and the definition of $a_{k}^{2}$, relation (1.7) yields the inequality $\left(1+b_{k+1}\right)>2 g_{2 k-1} g_{2 k}$. In a similar manner using (1.8) and (1.10), we obtain

$$
\left(1-b_{k+1}\right)>2\left(1-g_{2 k-1}\right) g_{2 k} .
$$

Adding these inequalities we have $g_{2 k}<1$. By (1.9) and (1.10) we have

$$
B_{k+1}(1)=2\left(1-g_{2 k}\right)\left(1-g_{2 k+1}\right) B_{k}(1)
$$

and

$$
B_{k+1}(-1)=2\left(1-g_{2 k}\right) g_{2 k+1} B_{k}(-1) .
$$

From (1.15), (1.17), (1.18) and our assumptions under the induction it follows that $g_{2 k},\left(1-g_{2 k}\right)\left(1-g_{2 k+1}\right)$, and $\left(1-g_{2 k}\right) g_{2 k+1}$ are positive. Hence $0<g_{2 k}<1$ and $0<g_{2 k+1}<1$, and the induction is complete.

The sufficiency part of the proof follows immediately from (1.9) and (1.10). It is important to notice that the formulas (1.9) and (1.10) hold only with $g_{0}=0$. The existence of such a sequence of minimal parameters will be discussed in $\S 2$.

Case 2. The $a_{p}^{2}$ nonnegative. In this case the determinants $B_{p}(1)$ and $B_{p}(-1)$ separate into blocks of nonoverlapping determinants. We suppose that $a_{1}^{2}, a_{2}^{2}, \cdots, a_{p-1}^{2}$ are positive and $a_{p}^{2}$ is zero. (This corresponds to the case of a terminating continued fraction.) It will be seen that the first $p$ forms in (1.3) and (1.4) are positive semidefinite if and only if $a_{1}^{2}, a_{2}^{2}, \cdots, a_{p-1}^{2}$ and $b_{1}, b_{2}, \cdots, b_{p}$ form a terminating double chain sequence. The proof is similar to Case 1. 
If a finite number of the $a_{p}^{2}$ are equal to zero, we simply repeat the argument in Case 2 for each finite block and the argument in Case 1 for the infinite block. If an infinite number of the $a_{p}^{2}$ are equal to zero, we repeat the argument of Case 2 for each finite block. This completes the proof.

We remark that Theorem 1.1 can be obtained by an inductive process using the theorem indicated at the beginning of the section [4]. This theorem says that the quadratic form

$$
\sum_{p=1}^{n} \beta_{p} x_{p}^{2}-2 \sum_{p=1}^{n-1} \alpha_{p} x_{p} x_{p+1}, \quad n=2,3,4, \cdots,
$$

is nonnegative if and only if $\beta_{n} \geqq 0$ and there exist numbers $h_{0}, h_{1}, \cdots$, such that $\alpha_{n}^{2}=\beta_{n} \beta_{n+1}\left(1-h_{n-1}\right) h_{n}, 0 \leqq h_{n-1} \leqq 1, n=1,2,3, \cdots$. In the proof of Theorem 1.1 by this method we apply the transformation

$$
h_{n}=\frac{g_{2 n-1} g_{2 n}}{g_{2 n-1} g_{2 n}+\left(1-g_{2 n}\right)\left(1-g_{2 n+1}\right)}, n=1,2,3, \cdots \quad\left(h_{0}=0\right),
$$

in (1.3) and

$$
h_{n}=\frac{\left(1-g_{2 n-1}\right) g_{2 n}}{\left(1-g_{2 n-1}\right) g_{2 n}+\left(1-g_{2 n}\right) g_{2 n+1}}, n=1,2,3, \cdots \quad\left(h_{0}=0\right)
$$

2. Jacobi fractions and moment sequences. In the theory of totally monotone sequences the monotone Hausdorff moment problem is solved [5]. A sequence of real numbers $\left\{c_{n}\right\}, n=0,1,2, \cdots\left(c_{0}=1\right)$, is said to be a monotone Hausdorff moment sequence if there exists a monotone nondecreasing real function $\phi(u), 0 \leqq u \leqq 1$, such that $c_{n}=\int_{0}^{1} u^{n} d \phi(u), n=0,1,2, \cdots$. It is shown that such a sequence is a monotone Hausdorff moment sequence if and only if the power series $\sum_{n=0}^{\infty} c_{n} x^{n}$ has a Stieltjes type continued fraction expansion of the form

$$
\frac{1}{1}-\frac{\left(1-g_{0}\right) g_{1} x}{1}-\frac{\left(1-g_{1}\right) g_{2} x}{1}-\cdots,
$$

where $0 \leqq g_{n} \leqq 1, n=0,1,2, \cdots$.

The continued fraction solution of the "extended" monotone Hausdorff moment problem has recently been given [3]. A sequence of real numbers $\left\{c_{n}\right\}, n=0,1,2, \cdots\left(c_{0}=1\right)$, is said to be an "extended" monotone Hausdorff moment sequence if there exists a monotone nondecreasing real function $\phi(u),-1 \leqq u \leqq 1$, such that $c_{n}$ $=\int_{-1}^{1} u^{n} d \phi(u), n=0,1,2, \cdots$. 
TheOREM 2.1. The sequence $\left\{c_{n}\right\}$ is an "extended" Hausdorff moment sequence if and only if the power series $\sum_{n=0}^{\infty} c_{n} x^{n}$ has a Jacobi type continued fraction expansion

$$
\frac{1}{1+b_{1} x}-\frac{a_{1} x^{2}}{1+b_{2} x}-\frac{a_{2} x^{2}}{1+b_{3} x}-\cdots,
$$

in which $\left\{a_{n}\right\},\left\{b_{n}\right\}$ form a double chain sequence.

We shall indicate how the theorem may be obtained by a transformation. It is well known that $P(z)=\sum_{n=0}^{\infty} c_{n} z^{n}$ is a moment generating function for the "extended" manotone Hausdorff moment problem if and only if $Q(w)=(1+z) P(z)$, where $w=(2 z /(1+z))$, is a moment generating function for the regular monotone Hausdorff moment problem.

Proof. By replacing $x$ by $2 x /(1+x)$ in the even part of $(2.1)$ and making an equivalence transformation we obtain a continued fraction of the type (2.2) in which the $a_{p}$ and $b_{p}$ are of the forms (1.5) and (1.6). Using the theorem stated at the beginning of the section the proof is immediate.

It is possible to extend several further results in the theory of ordinary chain sequences. One of these pertains to the minimal parameters of a chain sequence. In the proof of Theorem 1.1 the following result was used: A double chain sequence $\left\{a_{p}^{2}\right\},\left\{b_{p}\right\}$ has minimal parameters $l_{p}, m_{p}$, where $0 \leqq l_{p} \leqq g_{2 p-1}, 0 \leqq m_{p} \leqq g_{2 p}, p=0,1,2, \cdots$, given by

$$
l_{1}=\frac{1-b_{1}}{2}
$$$$
l_{p+1}=\left\{\begin{array}{l}
0 \quad \text { if } \quad m_{p}=1, \\
\frac{b_{p+1}-\left[1-2 m_{p}\left(1-l_{p}\right)\right]}{-2\left(1-m_{p}\right)} \text { if } \quad m_{p}<1,
\end{array}\right.
$$

$$
p=1,2,3, \cdots,
$$

$m_{0}=0$,

$$
m_{p+1}=\left\{\begin{array}{rr}
0 \text { if } m_{p}=1, \text { or } l_{p+1}=0 \text { or } 1, \\
\frac{a_{p+1}^{2}}{4\left(1-m_{p}\right) l_{p+1}\left(1-l_{p+1}\right)} \text { if } \quad m_{p}<1,0<l_{p+1}<1, \\
p=0,1,2, \cdots .
\end{array}\right.
$$

The proof of (2.3) and (2.4) follows by the methods of ordinary chain sequences. 
The minimal parameters of a double chain sequence have been shown to have a geometric representation in "extended" Hausdorff moment spaces [1], [2], [3].

By contractions and the methods for ordinary chain sequences expressions for the maximal parameters of a double chain sequence can be obtained. In addition to these formulas, theorems (11.2), (19.1), and (20.2) of [4] can also be extended by the same methods to the case of double chain sequences.

\section{REFERENCES}

1. S. Karlin and L. S. Shapley, Geometry of moment spaces, Mem. Amer. Math. Soc. No. 12 (1953), 93 pp.

2. F. Riesz, Sur certains systèmes singuliers d'equations intégrales, Ann. Sci. École Norm. Sup. (3) 28 (1911), 34-62.

3. Robert Seall and Marion Wetzel, Some connections between continued fractions and convex sets, Pacific J. Math. 9 (1959), 861-873.

4. H. S. Wall, Analytic theory of continued fractions, Van Nostrand, New York, 1948.

5. - Continued fractions and totally monotone sequences, Trans. Amer. Math. Soc. 48 (1940), 165-184.

ILLINOIS INSTITUTE OF TECHNOLOGY AND DENISON UNIVERSITY 